\title{
ON ASSESSMENT AND EVALUATION OF TEACHING COMPUTER NETWORKS TO ELECTRICAL ENGINEERING STUDENTS BY THE AID OF A LAB COURSE
}

\author{
Atef Abdrabou*iD, Walid Shakhatreh iD \\ UAE University, Electrical Engineering Dept., College of Engineering (United Arab Emirates) \\ *Corresponding author: atef.abdrabon@uaeu.ac.ae, walids@@uaeu.ac.ae
}

Received December 2020

Accepted May 2021

\section{Abstract}

In the era of the Internet-of-everything, learning the principles of data communications and networking is inevitable for many electrical engineering disciplines. The paper addresses the effectiveness of teaching the fundamentals of data communications and networking using a dedicated lab course as a co-requisite to a classic lecture-based course. In the introduced lab course, the students are asked to do a variety of tasks using real hardware and a network simulator. The paper introduces quantitative measures of an outcomebased learning approach applied to both courses. Based on students' achievements, the role of the lab course in the attainment of both the course learning outcomes and the electrical engineering program learning outcomes is measured in comparison with the case where the lab course is not taken. Our findings reveal a general enhancement trend in attaining the course and program learning outcomes with a significant increase in the program outcome related to solving engineering problems. Also, a slight increase is noticed in meeting the lab course outcomes for the students who attended the lab with the course in the same semester, which indicates an improvement in gaining practical knowledge.

Keywords - Learning, Outcome assessment, Teaching, Computer networks, Lab, Electrical engineering.

\section{To cite this article:}

Abdrabou, A., \& Shakhatreh, W. (2021). On assessment and evaluation of teaching computer networks to electrical engineering students by the aid of a lab course. Journal of Technology and Science Education, 11(2), 388-402. https://doi.org/10.3926/jotse.1186

\section{Introduction}

This digital era witnessed tremendous growth in the number of people who use the Internet for many purposes, such as e-commerce, information searching, infotainment, social networking, to name a few. Indeed, the ubiquitous Internet connectivity has changed the way we, as humans, live on earth. Moreover, new paradigms, such as Internet-of-things, machine-to-machine communication, fog, and cloud computing, are currently changing the way devices or machines operate.

Admittedly, electrical engineering (EE) undergrads represent a significant part of the future human driving force of this digital era with its continuous developments and advances. Thus, it is inevitable for EE undergrads to understand the basic principles of data communications and Internet operation to cope 
with the emerging evolutions such as Industry 4.0. Although many EE undergrad students these days are quite well engaged with social networking, a significant number of them have a lack of knowledge about the fundamentals of data communications and computer networks. Indeed, most electrical engineering curricula often contain a senior-level course about data communications and networking. However, introducing the theoretical concepts of this course without hands-on practice makes it difficult for the students to be engaged in a deep learning experience with a lifelong impact.

Thus, this paper focuses on teaching the foundations of data communications and networking for senior EE undergrad students using two compulsory courses; one is a classic lecture-based course, whereas the other is a dedicated lab course, following an outcome-based learning (OBL) approach. This approach allows the evaluation of the quality of the educational process based on evidence of students' achievements (Barkley \& Major, 2015; Carless, 2015). The evidence often includes data from selected assessment tools or tasks that the students are given throughout studying a specific course. These tasks should be carefully selected to check the level of students' involvement in the educational process, which, in turn, leads to enriching their lifelong learning experience (Fisher, Zeligman \& Fairweather, 2005; Carless \& Zhou, 2016; Mitchell \& Delaney, 2004). Besides, the right application of outcome-based learning requires course-level effective alignment between teaching, student pedagogical activities, assessment tools, and learning outcomes (El-Maaddawy \& Deneen, 2017). It also requires a suitable mapping between course learning outcomes (CLOs) and program learning outcomes (PLOs).

The programs of the Electrical Engineering department of the authors' institution are accredited by the Engineering Accreditation Committee of Accreditation Board for Engineering and Technology (ABET) according to clearly defined learning outcomes (ABET, 2012). Therefore, this paper aims at studying the impact of offering a dedicated lab course, as a co-requisite of a classic lecture-based course in data communications and networking, on the attainment of the learning outcomes of the classic course offering and the EE program outcomes. The contribution of this work is three-fold. First, it introduces a syllabus of a lab course designed to complement the offering of a regular course in data communications and networking for EE students. Besides the lab experiments, the course is enriched with assignments and network troubleshooting scenarios using a simulator tool that mimics the lab environment. Second, the paper offers a quantitative analysis for the assessment results of an OBL approach for the achievements of students who attended only the lecture-based course compared with the results of the students who concurrently attended the lab also. Third, the paper quantitatively measures the impact of offering the lab course on meeting the EE program learning outcomes following the ABET criteria (ABET, 2012).

In the rest of the sections, the paper offers a review of the most relevant research works and then portrays the study context. After that, the research methodology is presented in detail, including the participants, data collection, and analysis. The results are then introduced and discussed. Finally, the paper is concluded.

\section{Background}

In the literature, various research works propose to add lab experiments to computer networks courses. In one research work, a methodology is proposed for reforming a fundamental course about computer networks offered with lab experiments (Gao, Yao, Yao, Cui \& Zhang, 2009). Similarly, Zhang and Xiong (2013) address setting lab experiments as a part of computer networks' courses in local universities. However, these works (Gao et al., 2009; Zhang \& Xiong, 2013) do not apply OBL, and the effectiveness of the proposed methods is neither measured nor provided. Sarkar (2006) offers a detailed description of laboratory experiments for a course about networking and telecommunications. The experiments are designed to be practical exercises of the lecture-based course, not a separate lab course (Sarkar, 2006). Although the CLOs are mentioned, the proposed lab exercises are indirectly evaluated by a feedback survey and students' grades, but the impact on CLOs and PLOs is not measured. Other research works suggest providing lab experiments remotely over the Internet with a lecture-based course about computer networks (Vivar \& Magna, 2008). The work presents a comparison between the grades of the students who accessed the lab experiments remotely and those who have direct access. However, the impact of introducing remote lab experiments on learning outcomes is not provided (Vivar \& Magna, 2008). 
Another work proposes giving students failure case studies to investigate in a lab that accompanies the lecture of a course about computer network engineering (Yu \& Zhang, 2010) but without providing an evaluation of the proposed method.

On the other hand, several research works introduce integrating network simulator or emulator tools to support the lecture-based teaching of computer networks. Some of these works propose using virtualization-based network emulator tools to provide virtual lab experiments or exercises without using hardware (Gurgel, Barbosa \& Branco, 2013; Jayalaxmi, Suvarna \& Sujatha, 2012). Other research works introduce the usage of computer games offered by Cisco Academy in teaching computer networks (Zhamanov \& Sakhiyeva, 2015). Nevertheless, the authors of these works do not provide a structure for a separate lab course. Also, they do not evaluate the impact of the proposed tools on the attainment of learning outcomes. Vijayalakshmi, Desai and Raikar (2017) introduce an OBL approach to teach computer networks using simulation software (Packet Tracer). Some course outcomes are evaluated before and after Packet Tracer activities. However, no lab experiments are included.

A few research works in the literature propose offering a separate lab course in addition to a regular course to teach computer networks. One work introduces a lab course with a description set of experiments for third-year undergrad students and first-year graduate students (Liu, Zhang \& Jiao, 2009). Another research presents a work-in-progress about offering an elective lab course (Gao, Peng, Yin, Hei \& Wang, 2018). The authors of these works do not evaluate the proposed course based on the degree of attainment of learning outcomes.

To the best of our knowledge, no other research work in the literature provides the contents of a lab course and a quantitative evaluation of the impact of offering it as a co-requisite to a classic course in computer networks following an OBL approach.

\section{Study Context}

This section presents the subjects covered by the Data Communications and Networking and Data Communications and Networks Laboratory courses that are regularly offered to senior students in the Electrical Engineering (EE) department of UAE University.

The two courses are compulsory courses for the students of the two available programs in the EE department, namely, Electrical Engineering and Communication Engineering programs. In the OBL approach, every course outcome should be mapped to at least one program outcome. The program learning outcomes (PLOs) addressed by both courses are introduced in Table 1. The program learning outcomes are generally set to be broader in scope than course learning outcomes as they are used to measure the realization of the objectives of an entire program. These outcomes follow the ABET guidelines (ABET, 2012). Admittedly, the quantitative measuring of students' achievements in both courses reflects directly on the attainment of the EE department programs' outcomes. This, in turn, influences achieving the overall scholarly objectives of UAE University as an academic institution. Thus, the following subsections present the course learning outcomes (CLOs) for both courses and how they are mapped to the PLOs.

\begin{tabular}{|l|l|}
\hline \multicolumn{1}{|c|}{ PLOs } & \multicolumn{1}{c|}{ Description } \\
\hline First & Ability to design and conduct experiments safely, as well as to analyze and interpret data. \\
\hline Second & $\begin{array}{l}\text { Ability to design electrical (and communication) components, systems, or processes to meet desired } \\
\text { specifications and imposed constraints such as economic, environmental, social, political, ethical, health } \\
\text { and safety, manufacturability, and sustainability. }\end{array}$ \\
\hline Third & $\begin{array}{l}\text { Ability to identify, formulate, and solve problems encountered in electrical (and communication) } \\
\text { engineering practice. }\end{array}$ \\
\hline Fourth & $\begin{array}{l}\text { Ability to use the techniques, skills, and modern engineering tools necessary for electrical and } \\
\text { communication engineering practice. }\end{array}$ \\
\hline
\end{tabular}

Table 1. The program learning outcomes addressed by the lecture-based and laboratory-based courses 


\subsection{Data Communications \& Networking Course: Subjects and CLOs}

The Data Communications and Networking course accounts for 3 credit hours in the curricula of the electrical and communication engineering programs. The course is offered in two 75-minute lectures per week over 16 weeks covering the fundamentals of information transfer via computer networks and their applications.

Therefore, it starts by introducing the open system interconnect (OSI) model then presents the network access (physical and data link) layers in detail, including typical topics such as transmission media, multiplexing with analog and digital transmissions, local and wide area networks (LANs and WANs), medium access control, multiple access, error detection and correction with a focus on the corresponding IEEE standards. It also addresses the operation of networked applications over the TCP/IP stack with topics such as network layer addressing and subnetting, transport layer flow and congestion control mechanisms, client-server and peer-to-peer models. The course learning outcomes that the students should have gained by the end of the course are shown in Table 2 .

\subsection{Data Communications and Networks Lab Course: Subjects and CLOs}

The Data Communications and Networks Laboratory course is conducted as lab sessions of two hours and a half of contact time for each. The course is introduced over 15 weeks. Each week contains one lab session, and two weeks are dedicated to the final and midterm exams. The majority of the laboratory experiments are divided into two sessions. The students are asked to conduct the experiment in the lab, replicate the experiment with modifications using the Packet Tracer simulator, and write a lab report describing their observations regarding the hardware and simulated experiments. The Packet Tracer simulator imitates the real lab equipment with a simulated library of Cisco network components that can be configured exactly the same way as real hardware. Moreover, the simulator allows packet tracing in a user-friendly manner (Janitor, Jakab \& Kniewald, 2010). Figures 1(a) and 1(b) show the laboratory equipment, whereas Figure 2 shows the base configuration used in the Packet Tracer simulator.

\begin{tabular}{|c|c|c|}
\hline Lecture Course CLOs & PLOs & Description \\
\hline CLO-1 & PLO-3 & $\begin{array}{l}\text { Recognize application layer protocols, open systems interconnection } \\
\text { (OSI) reference model, TCP/IP architecture model. }\end{array}$ \\
\hline CLO-2 & PLO-3 & $\begin{array}{l}\text { Identify different transmission media and the fundamental } \\
\text { limits of digital transmission. }\end{array}$ \\
\hline CLO-3 & PLO-3 & $\begin{array}{l}\text { Apply the coding techniques that can be used to detect and } \\
\text { correct errors that may occur during digital transmission. }\end{array}$ \\
\hline CLO-4 & PLO-3 & $\begin{array}{l}\text { Identify the multiplexing techniques used to share transmission } \\
\text { media. }\end{array}$ \\
\hline CLO-5 & PLO-3 & $\begin{array}{l}\text { Identify transport layer protocols; namely, user datagram } \\
\text { protocol (UDP) and transport control protocol (TCP). }\end{array}$ \\
\hline CLO-6 & PLO-3 & $\begin{array}{l}\text { Recognize the standard data link layer protocols that provide } \\
\text { reliable data transfer service. }\end{array}$ \\
\hline CLO-7 & PLO-3 & $\begin{array}{l}\text { Identify the different multiple access techniques that reduce } \\
\text { collision and interference from other users; namely, random access, } \\
\text { scheduling, and channelization. }\end{array}$ \\
\hline CLO-8 & PLO-3 & $\begin{array}{l}\text { Identify current network technologies such as Ethernet, } \\
\text { wireless LANs, and Bluetooth. }\end{array}$ \\
\hline CLO-9 & PLO-2, PLO-3 & $\begin{array}{l}\text { Create Internet Protocol (IP) sub-networks, and static routing } \\
\text { tables. }\end{array}$ \\
\hline CLO-10 & PLO-3 & $\begin{array}{l}\text { Differentiate between connecting devices such as hubs, } \\
\text { bridges, switches, and routers. }\end{array}$ \\
\hline
\end{tabular}

Table 2. The course learning outcomes for the lecture-based course mapped to the program learning outcomes 


\subsubsection{Experiment 1: Lab Devices}

This laboratory is divided into two sessions. The first session is introductory to make the students get acquainted with the different types of network devices in the lab, their functions, configurations, and basic network commands. In this session, the students first learn how to check the configuration of the lab PCs. After that, the students are introduced to different lab devices, such as Ethernet switches and routers, and their main internal components. They are also asked to issue simple configuration commands directly by terminal access through the console port and remotely by using Telnet. The second session aims to introduce the Packet Tracer simulator. The students are asked to use the same lab components to make a simple network using the configuration commands they learned with hardware. By completing this experiment, the students should be able to describe the lab devices, their functions, and components. They should also be able to issue basic configuration commands, save, and restore configuration files.

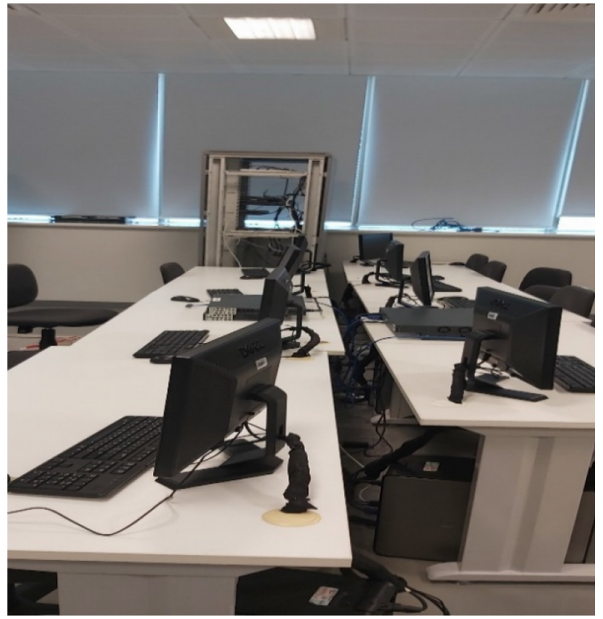

(a) Some of the LAN computers and WAN routers of the lab setup.

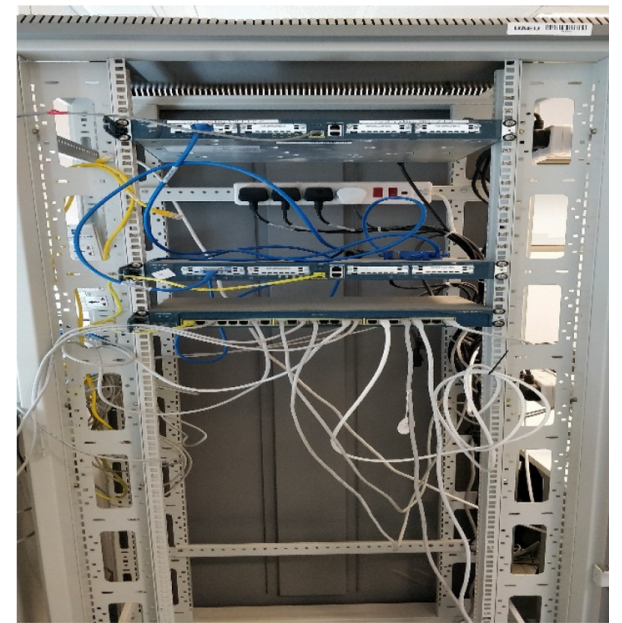

(b) The LAN connections during a lab session.

Figure 1. The computer networking laboratory setup

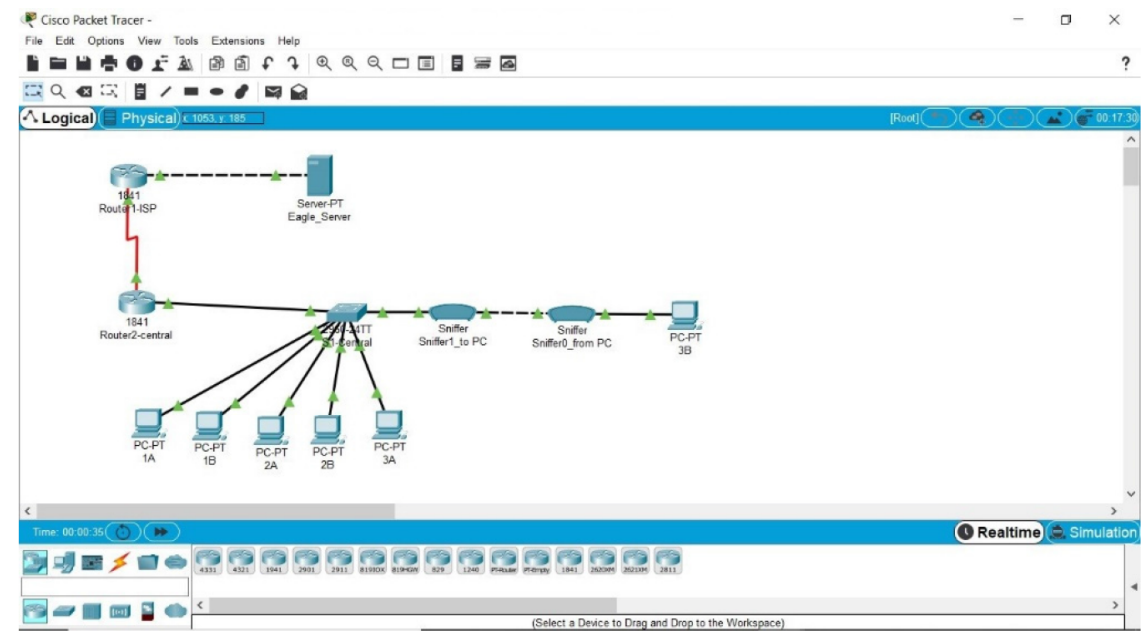

Figure 2. The Packet Tracer base configuration 


\subsubsection{Experiment 2: Local Area Network Cabling}

This experiment represents a practical example of the Physical layer topic. During the lab session, the students are asked to check the different cable types used in local area networks. The steps to make an Ethernet cable by attaching two RJ-45 terminal connectors to the right wires are introduced to the students in two configurations (straight-through and crossover). The students are asked to prepare their own Ethernet cables and test them first using a cable tester then by connecting them to a computer. By the end of this experiment, the students should be able to recognize different cable types, make working Ethernet cables, and test them.

\begin{tabular}{|c|c|l|}
\hline Laboratory Course CLOs & \multicolumn{1}{|c|}{ PLOs } & \multicolumn{1}{|c|}{ Description } \\
\hline CLO-1 & PLO-1, PLO-4 & $\begin{array}{l}\text { Investigate data communication basics and how networks work } \\
\text { focusing on network architectures, components, scalability, and } \\
\text { troubleshooting tools. }\end{array}$ \\
\hline CLO-2 & PLO-1, PLO-4 & $\begin{array}{l}\text { Investigate basic functions and types of network devices, } \\
\text { media, and protocols that enable network communication. }\end{array}$ \\
\hline CLO-3 & PLO-1, PLO-4 & $\begin{array}{l}\text { Investigate OSI model and its layers, their roles and functions, } \\
\text { standards, and protocols. }\end{array}$ \\
\hline CLO-4 & PLO-1, PLO-4 & $\begin{array}{l}\text { Explore the concepts of routing packets related to addressing, path } \\
\text { determination, data packets, and IP. }\end{array}$ \\
\hline CLO-5 & PLO-1, PLO-4 & $\begin{array}{l}\text { Analyze network addressing and how to use the network mask, or } \\
\text { prefix length, to determine the number of subnetworks and hosts in } \\
\text { a network. }\end{array}$ \\
\hline CLO-6 & PLO-1, PLO-4 & $\begin{array}{l}\text { Explore the basic concepts of encapsulation processes that } \\
\text { occur as data travels across a LAN and a WAN. }\end{array}$ \\
\hline CLO-7 & PLO-1, PLO-4 & $\begin{array}{l}\text { Utilize simulation tools using Packet tracer and Wireshark software } \\
\text { to practice IP subnetting, building Planning and configuring a } \\
\text { complex network. }\end{array}$ \\
\hline
\end{tabular}

Table 3. The course learning outcomes for the lab course mapped to the program learning outcomes

\subsubsection{Experiment 3: IP Addressing and Subnetting}

The experiment focuses on the fundamentals of Internet protocol (IP) addressing, including classful and classless addressing. It targets designing IP subnetworks from a given address space with variable network masks based on user demand. The experiment consists of two sessions. In the first session, the students practice several exercises on IP subnetting. In the second session, the students are requested to design subnetworks for an organization based on a specific demand for IP addresses. Moreover, the students are requested to assign the calculated IP addresses to a pre-connected network in the Packet Tracer software tools using the configuration commands they learned in Experiment 1. They are also asked to write the contents of a static routing table. By the end of this experiment, the students should be able to develop an efficient design of subnetworks (in terms of the number of IP addresses) from a given IP address space based on the user demand.

\subsubsection{Experiment 4: Building a Wide Area Network}

In this experiment, the students apply their knowledge from the previous experiments and the lectures to build a WAN from scratch using the lab equipment and the Packet Tracer software. The students are asked to (i) define the required network devices, (ii) design appropriate IP subnetworks on-demand from the given address space, (iii) configure router interfaces and PCs with the calculated IP addresses, (iv) build static routing tables, (iv) test the configuration, and (v) document the results. The experiment is performed in two sessions. One session is dedicated to building the WAN using lab equipment, whereas, in the other session, the students work on building a bigger WAN using the Packet Tracer application. By the end of the experiment, the students should be able to design, connect, and configure a WAN connecting several remote sites. 


\subsubsection{Experiment 5: LAN Configuration and Troubleshooting}

This experiment targets local area networks and other related topics such as troubleshooting tools and the address resolution protocol (ARP) over two sessions. In one session, the students are asked to use the ping command and capture the contents of its messages using the Wireshark software. They should also observe the structure of the Internet control message protocol (ICMP) packets for query and error-reporting messages. The students should also discover the role of the ARP protocol by observing the Wireshark captures of its messages. The experiment also addresses the usage of other troubleshooting tools like traceroute. In the other session, the students explore the operation of LAN switches. They configure the switch using Telnet and observe the operation of its address table. Besides, the students capture the Ethernet frame and check its contents and error detection code. By the end of this experiment, the students should be able to use different troubleshooting tools, describe the role of the ARP protocol, issue basic configuration commands of an Ethernet switch, and distinguish the Ethernet frame contents.

\subsubsection{Experiment 6: TCP and UDP Traffic}

In this experiment, the students learn how connection-oriented and connectionless services are provided in the transport layer by observing the operational procedure of the transmission control protocol (TCP) and user datagram protocol (UDP). The students are asked to make their observations using the netstat tool and by exploring the Wireshark captures of TCP and UDP traffic focusing on the TCP and UDP headers. Furthermore, they observe the TCP protocol's role in establishing a reliable connection for basic applications' traffic such as web browsing and file transfer. The students also use the Packet Tracer simulator to observe the packet capture of an end-to-end connection of a network configuration of a larger scale than the lab network. By the end of this experiment, the students should be able to recognize the difference between the two transport layer protocols commonly used over the Internet (TCP and UDP) in terms of operation, header contents, and the type of applications that each one serves.

\subsubsection{Experiment 7: Client/Server Applications}

This experiment focuses on the client-server model of the application layer. The students are asked to install the server-side for different applications, such as web browsing and email. The configuration of the server-side is one of the essential requirements of this experiment. Moreover, the students are requested to analyze the HTTP and email traffic between the client and server using Wireshark capture. The students should record the different messages exchanged between the client and server and analyze their contents. By the end of this experiment, the students should be capable of performing the basic configuration of a web server and an email server. They should be able to describe the interaction between the client and server for a request-response application such as web browsing and a one-way transaction application such as email.

\section{Methodology}

This research tackles the following questions (i) does teaching computer networks to electrical engineering students using a dedicated lab course in addition to the classic lecture-based course impact the attainment of the learning outcomes of the courses and program? (ii) how the performance of electrical engineering students is affected if they take the computer networks lab course with or after the lecture-based course?

\subsection{Participants}

In order to address the research questions, two courses in the curriculum of the electrical engineering program are investigated, namely, the "Data Communications and Networking" (a lecture-based course) and "Data Communications and Networks lab". The assessment data of these courses are collected over a period of four years. The first author taught the Data Communications and Networks course, and the second author taught the lab course during this period. The two compulsory courses are attended by 170 male and female students over the study period. Out of this number, 133 students took the lab course 
with the lecture-based course in the same semester, whereas the rest attended the lab in a different semester from the course. All the students have the same profile. There is no academic-related reason preventing some students from taking the two courses in the same semester. Some students take the lab in a different semester from the course only to have a more comfortable class schedule since labs are usually offered late afternoon while the lectures are typically offered from morning till afternoon. The number of students who took the courses under consideration during the study period is $36,40,47,47$ for the Years 2014, 2015, 2016, and 2017, respectively.

\subsection{Data Collection}

The performance of the students should be carefully assessed to address the research questions using the OBL approach. The evaluation of the students' achievements for the lecture-based course and lab course under investigation is performed by different assessment tasks, as summarized in Table 4 and Table 5, respectively. For the lecture-based course, the assessment tasks were biweekly short quizzes, two progress tests (each one is done in one part of the semester), midterm and final exams. The questions of each exam are typical theoretical computer networking questions, which are mapped to the course CLOs. The mapping is written before the question statement. For the lab course, the assessment tasks are more comprehensive to ensure that the students are getting the maximum benefit from the lab sessions. The tasks consist of pre-lab exercises to test the necessary background knowledge that the student should have to conduct the experiment steps effectively. During the lab sessions, the students work in groups. The group work is assessed by oral questions (during the experiment) and short quizzes (by the end of the lab session). The students are asked to write their observations and submit them in lab reports to be delivered within a specific due date after each experiment. A midterm and a final exam are also conducted to measure the students' overall attainment and abilities to link the information they gained across multiple experiments. Generally, each CLO for the lecture-based course or the lab course is assessed by at least two assessment tasks. By the end of the semester, the students' grades are collected, and the achieved results are calculated based on the method described in the Data Analysis section.

\begin{tabular}{|c|c|c|c|c|}
\hline Lecture-based Course CLOs & Quizzes & Progress Tests & Midterm Exam & Final Exam \\
\hline CLO-1 & $\checkmark$ & $\checkmark$ & $\checkmark$ & - \\
\hline CLO-2 & $\checkmark$ & $\checkmark$ & $\checkmark$ & $\checkmark$ \\
\hline CLO-3 & $\checkmark$ & $\checkmark$ & $\checkmark$ & - \\
\hline CLO-4 & - & $\checkmark$ & - & $\checkmark$ \\
\hline CLO-5 & $\checkmark$ & $\checkmark$ & $\checkmark$ & $\checkmark$ \\
\hline CLO-6 & $\checkmark$ & $\checkmark$ & $\checkmark$ & $\checkmark$ \\
\hline CLO-7 & $\checkmark$ & $\checkmark$ & $\checkmark$ & $\checkmark$ \\
\hline CLO-8 & $\checkmark$ & $\checkmark$ & - & $\checkmark$ \\
\hline CLO-9 & $\checkmark$ & $\checkmark$ & $\checkmark$ & $\checkmark$ \\
\hline CLO-10 & $\checkmark$ & $\checkmark$ & $\checkmark$ & $\checkmark$ \\
\hline
\end{tabular}

Table 4. The assessment tasks of the lecture-based course mapped to the course learning outcomes

\begin{tabular}{|c|c|c|c|c|c|c|}
\hline Lab Course CLOs & Prelab Quest. & Oral Quest. & Quizzes & Lab Reports & Midterm Exam & Final Exam \\
\hline CLO-1 & $\checkmark$ & $\checkmark$ & $\checkmark$ & $\checkmark$ & $\checkmark$ & $\checkmark$ \\
\hline CLO-2 & $\checkmark$ & $\checkmark$ & $\checkmark$ & $\checkmark$ & $\checkmark$ & $\checkmark$ \\
\hline CLO-3 & $\checkmark$ & $\checkmark$ & $\checkmark$ & $\checkmark$ & - & $\checkmark$ \\
\hline CLO-4 & $\checkmark$ & $\checkmark$ & $\checkmark$ & $\checkmark$ & - & $\checkmark$ \\
\hline CLO-5 & $\checkmark$ & $\checkmark$ & $\checkmark$ & $\checkmark$ & - & $\checkmark$ \\
\hline CLO-6 & $\checkmark$ & $\checkmark$ & $\checkmark$ & $\checkmark$ & - & $\checkmark$ \\
\hline CLO-7 & $\checkmark$ & $\checkmark$ & $\checkmark$ & $\checkmark$ & $\checkmark$ & $\checkmark$ \\
\hline
\end{tabular}

Table 5. The assessment tasks of the lab course mapped to the course learning outcomes 


\subsection{Data Analysis}

The collected students' grades by the end of the semester are analyzed for each course outcome based on Table 4 and Table 5 for the lecture-based course and the lab course, respectively. The achievement percentage per CLO $i$ for the task $t$ of the student $k, A_{t k}^{i}$, is averaged over the number of students $N$ to obtain the percentage average students' attainment for task t per CLO $i, E A_{t}^{i}$ as

$$
E A_{t}^{i}=\frac{1}{N} \sum_{k=1}^{N} A_{t k}^{i}
$$

After that, the percentage overall students' attainment per CLO $i, S A^{i}$, is calculated from (1) by averaging $E A_{t}^{i}$ over the total number of tasks per CLO $i, M^{i}$, as

$$
S A^{i}=\frac{1}{M^{i}} \sum_{t=1}^{M^{i}} E A_{t}^{i}
$$

The achievements of the students for a CLO $i$ are considered satisfactory if they meet a target of $S A^{i}>70 \%$.

The CLO attainment impact on a PLO $j, P A$, is obtained by the aid of Table 2 and Table 3 by averaging $S A^{i}$ over the number of CLOs, $C_{j}$, that map to the PLO $j$ as in

$$
P A^{j}=\frac{1}{C_{j}} \sum_{i=1}^{C_{j}} S A^{i}
$$

When two CLOs map to the same PLO, each contributes equally to the attainment of the PLO. The students' achievement target for each PLO has been set to 70\%. Thus, if $P A^{j} \geq 70 \%$ for PLO $j$; this implies the target is met.

\section{Results}

This section introduces the impact of taking a lab course in computer networking by electrical engineering students in parallel with studying a classic lecture-based course in the same subject on the attainment levels of the CLOs of both courses. It also presents the effect of taking the lab course while studying the lecturebased course on achieving the learning outcomes of the electrical engineering program (PLOs). The results are obtained based on the assessment outcomes of the followed OBL approach for the courses "Data Communications and Networking" and "Data Communications and Networks Laboratory".

\subsection{Impact on CLO Attainment}

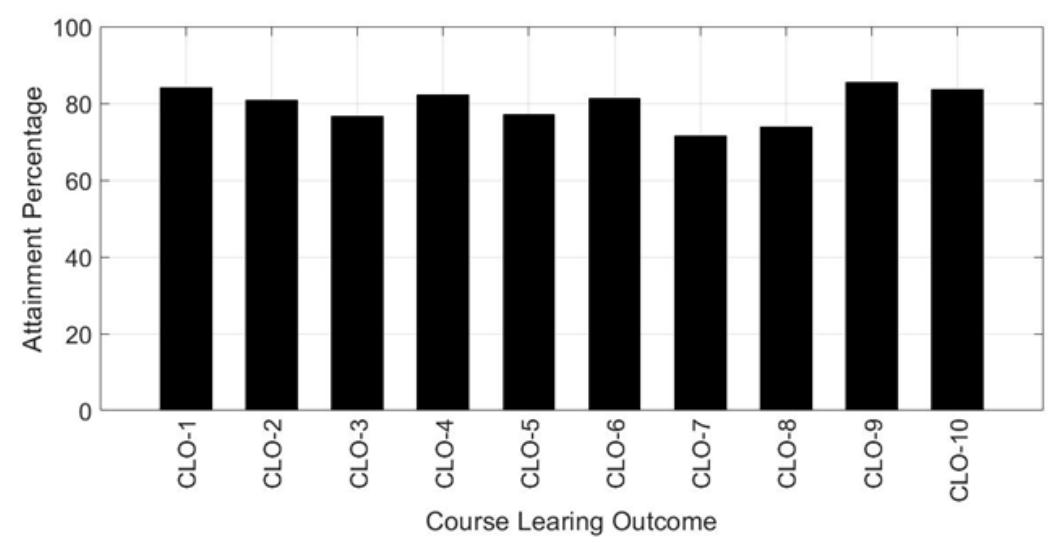

Figure 3. Attainment percentage of the lecture-based course CLOs for students also taking the lab course

Figure 3 shows the attainment percentage of the learning outcomes of the lecture-based course as achieved by the students who also attend the lab course. On the other hand, Figure 4 presents the attainment percentage per CLO for the students who only take the lecture-based course without the lab 
course. Apparently, as Figures 3 and 4 reveal, the achievements of the students who attend both courses outperform the students who attend the lecture-based course without the lab course in every CLO. This implies that offering a dedicated lab course in computer networks in addition to classic lecturing has a clear impact on the performance of electrical engineering students as the OBL approach mainly measures students' achievements.

Figures 5 and 6 present the attainment percentage of the lab course CLOs achieved by students who take the lecture-based course in parallel with the lab course and the students who take the lab course after, respectively. The figures reveal a slight improvement in the attainment percentage of the lab course CLOs, except for CLO-2, which depends on theoretical knowledge mainly studied in the lecture-based course. This indicates that the timely practical application of the theoretical knowledge acquired by lecturing increases the effectiveness of offering a lab course in computer networks to electrical engineering students.

\subsection{Impact on PLO Attainment}

Following an OBL approach helps to map the courses' CLOs to the program learning outcomes. This allows to easily extend the CLO-related research results (portrayed in the previous section) to the learning outcomes of the program that the electrical engineering students study.

The attainment of the relevant Electrical Engineering program PLOs (PLO-2 and PLO-3) of the lecturebased course is shown in Figure 7 for the students who attended the course with and without the lab course.

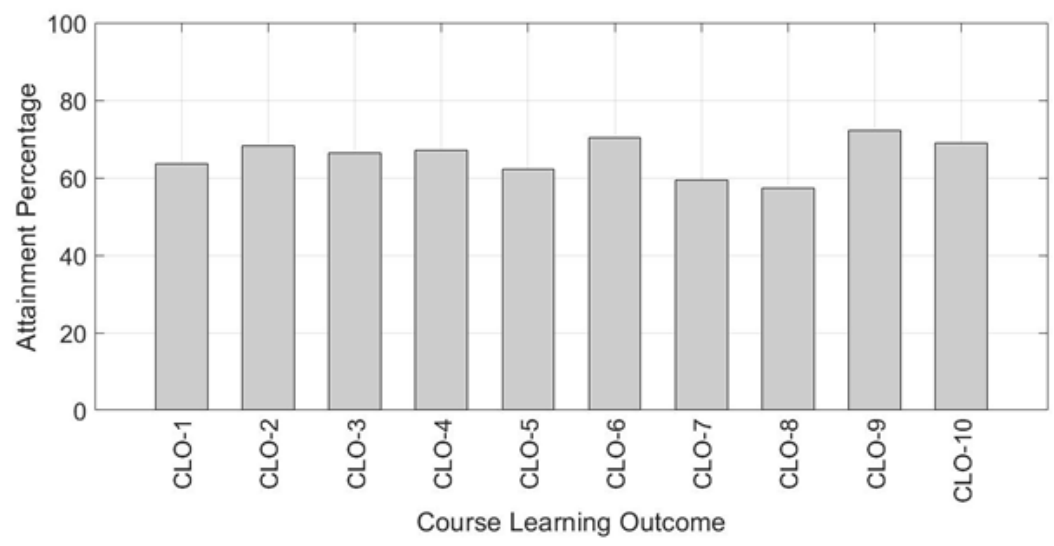

Figure 4. Attainment percentage of the lecture-based course CLOs for students not taking the lab course

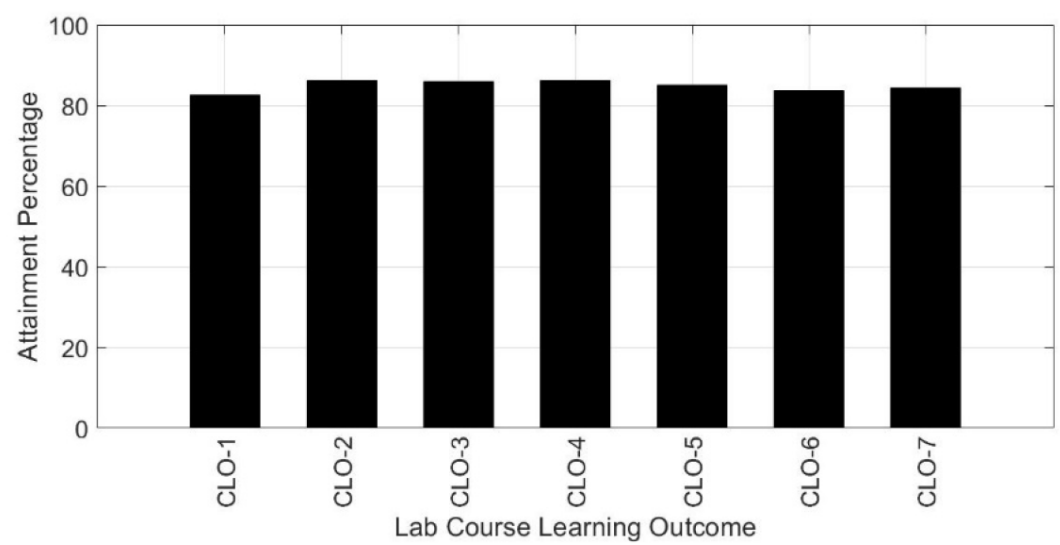

Figure 5. Attainment percentage of the lab course CLOs for students also taking the lecture-based course 
The figure reveals a clear improvement in the attainment percentage of both PLOs for the students who attended the lecture-based course with the lab course. Enhancing the PLOs targeted by the classic theoretical networking course is an indirect outcome to increasing the hands-on experience of electrical engineering students, who are looking forward to enhancing their practical skills to compete in the job market after graduation.

The attainment percentage of the PLOs related to the lab course (PL0-1 and PL0-4) is depicted in Figure 8. The figure compares the achievements of the students who attended the lab course and the lecture-based course in the same semester with those who attend the lab course in a subsequent semester. Figure 8 shows that target is met in both cases. However, the attainment percentage is slightly higher when the students attend both courses in the same semester.

Figure 9 present the percentage attainment of the PLOs related to the lecture-based course according to the grades obtained by $60 \%$ of the students who attended it with the lab course and without the lab course. The attainment target is considered satisfactorily met if $60 \%$ of the students scored $70 \%$ or above. The results revealed by Figure 9 indicate that only the students who take both courses in the same semester met the target, reflecting a better understanding of the theoretical course material.

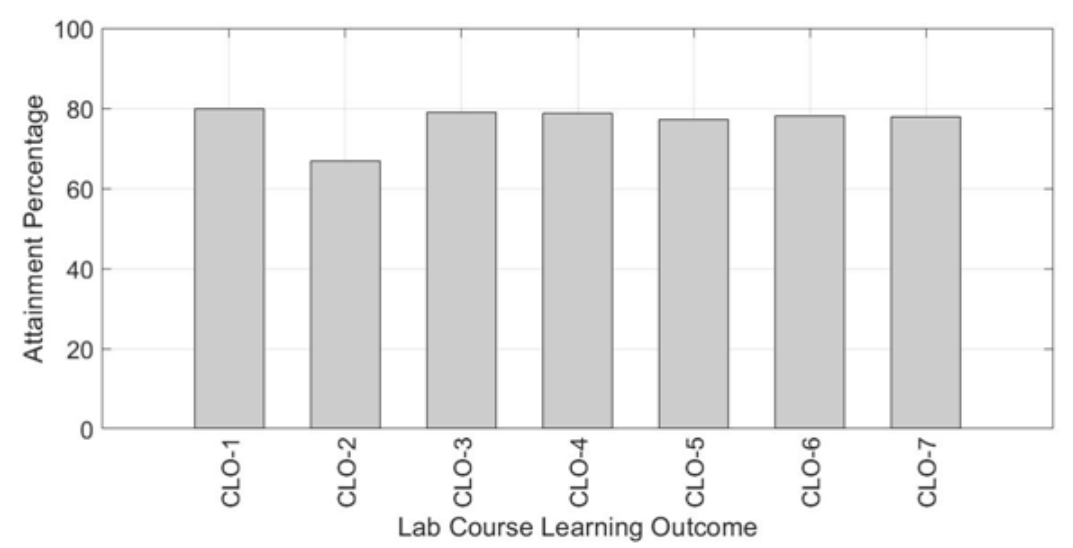

Figure 6. Attainment percentage of the lab course CLOs for students not taking the lecture-based course

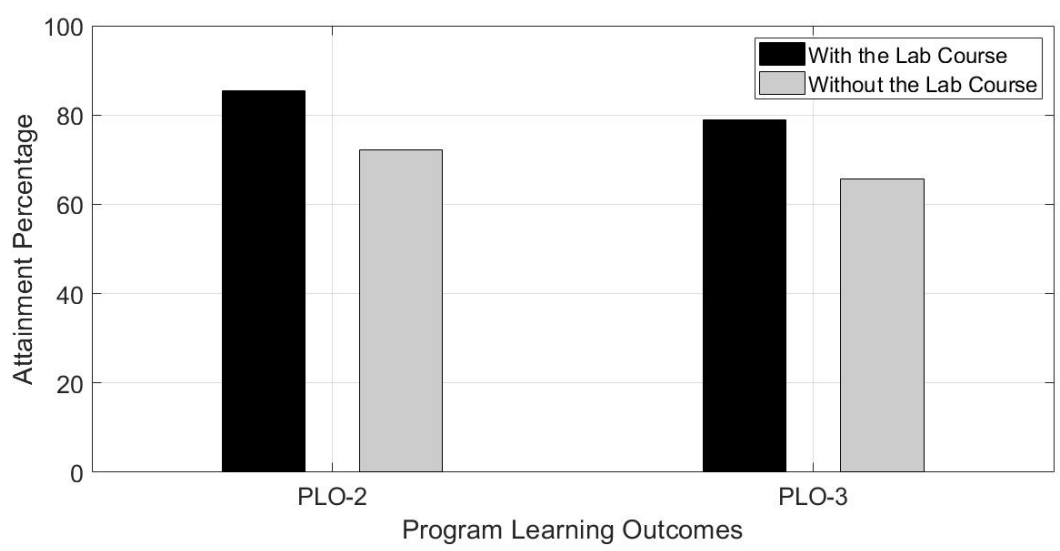

Figure 7. Percentage PLO attainment for the lecture-based course 


\section{Discussion}

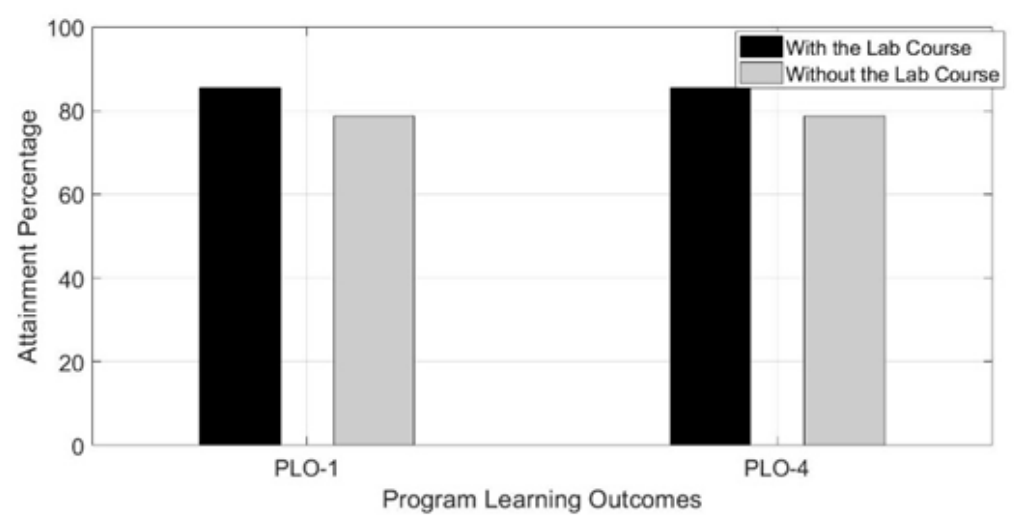

Figure 8. Percentage PLO attainment for the lab course

The results introduced in the previous section indicate a significant improvement in the average students' achievements for the lecture-based course CLOs, over the considered assessment tools whenever the lab course is attended in conjunction with it. Moreover, as Figure 4 reveals, only two CLOs meet the 70\% target for the students who do not attend the lab course in the same semester as the lecture-based course. On the other hand, a little impact is observed on students' performance for the lab course CLOs, as revealed in Figures 5 and 6, except for the CLO targeting background information about network devices and protocols (CLO-2), where the enhancement is significant. The reason for this trait is that the students attend the lab course while taking or after they take the lecture-based course. Thus, they are taught the theoretical foundations either way. However, the theoretical knowledge they gained from the lecture-based course helped them to better deal with network devices in the lab. The slight enhancement in their lab course achievements is pertained to applying the theoretical concepts in the laboratory experiments without delay, which contributes to improving their visual learning experience.

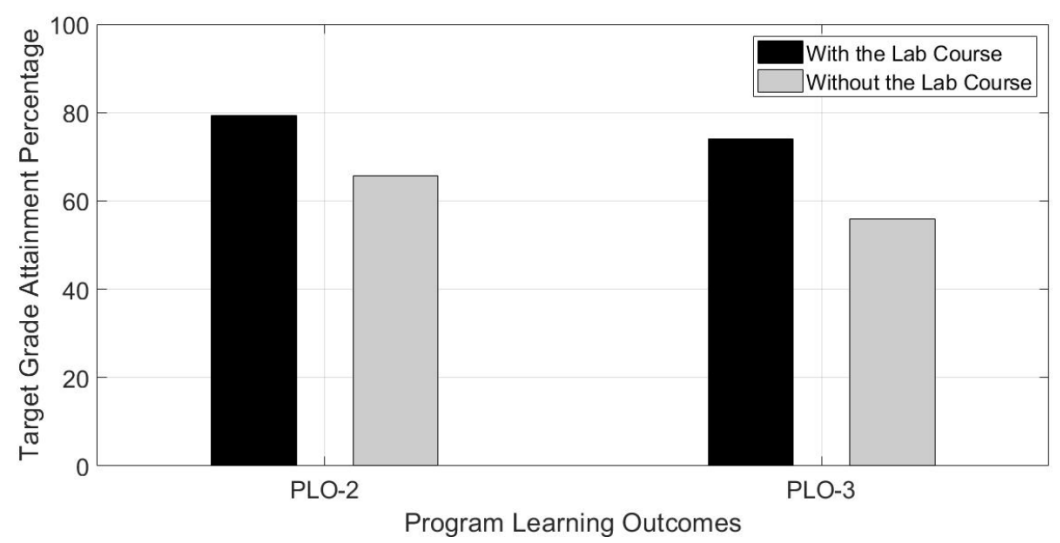

Figure 9. Percentage PLO attainment based on students' grades for the lecture-based course

Moreover, as shown in Figure 7, attending the lab course with the lecture-based course not only contributes to increasing the attainment percentage of the PLOs addressed by the theoretical course but also helps to meet the PLOs' attainment target for it. The figure depicts that the students were able to achieve the target of PLO-3 with the help of the lab course hands-on experience they gained. Besides, the students were able to generally enhance their grades in the theoretical course by attending the lectures and the lab sessions in the same semester, as revealed in Figure 9, since the students are able to see the practical applications of what they learned in the lecture-based course. This helps them reflect on how their deep understanding of theoretical concepts is valuable in real practice. 
On the other hand, the targets of the lab course-related PLOs are met irrespective of whether the students attend the lab sessions while taking the lectures or in a subsequent semester. This is due to the fact that PLOs addressed by the lab course are normally dependent on the lab activities and the students attend the lab after being taught the required theoretical background.

Although the paper targets teaching the computer networks subject to electrical engineering students, other subjects can benefit from the same experience as long as they target real systems that can be implemented or fully emulated in a university laboratory setting. For instance, students studying a course in embedded systems can benefit from taking a dedicated lab course in the same subject where they can apply the theoretical knowledge related to design the hardware and operating system software of an embedded computing system. Similarly, a course in wireless mobile communication can be taught in conjunction with a separate lab course where the lab contains real open-source cellular systems such as long-term evolution (LTE) fourth-generation (4G) system. Currently, these systems are easy to be built in a lab environment (Software Radio Systems Limited, 2021), thanks to the current advances in the processing capabilities of personal computers and the availability of affordable software-defined radio hardware.

\section{Conclusion}

The paper introduces the contents of a lab course in computer networks and presents a study of the impact of teaching a theoretical course in computer networks to electrical engineering students in conjunction with this lab course using an OBL approach. The structure and the topics of the lecture-based course are provided. In addition, the laboratory/simulation experiments practiced in the lab course sessions are offered in detail. The students' achievements over four years are measured using specific assessment tools that quantify the attainment of well-defined course learning outcomes for the lecture-based and lab courses. The course learning outcomes are linked and mapped to the electrical engineering program outcomes.

The results obtained from students' achievements of the lecture-based course reveal the effectiveness of delivering the theoretical aspects of computer networks to electrical engineering students by lecturing a classic course in addition to teaching them a dedicated lab course offering hands-on and simulation experience. Quantifying the students' achievements using an OBL approach indicates that attending the lecture-based course and the lab course in the same semester increases the attainment percentages of all the lecture-based course CLOs making them meet or exceed the specified target. Moreover, offering both courses in parallel improves the students' grades of the theoretical course, demonstrating a better understanding of the course material. In addition, it enhances the way the students use the network devices in the lab as they have a clearer understanding of how they operate. Furthermore, the lab course offering helps attain the electrical engineering program learning outcomes related to solving engineering problems since it compliments the students' theoretical knowledge by practical experience.

\section{Declaration of conflicting interests}

The authors declare that there is no conflict of interest.

\section{Funding}

The authors received no financial support for the research, authorship, and/or publication of this article.

\section{References}

ABET (2012). Available at: https://www.abet.org/wp-content/uploads/2015/04/etac-criteria-2013-2014.pdf

Barkley, E.F., \& Major, C.H. (2015). Learning assessment techniques: A handbook for college faculty. John Wiley \& Sons. 
Carless, D. (2015). Excellence in university assessment: Learning from award-winning practice. Routledge. https://doi.org/10.4324/9781315740621

Carless, D., \& Zhou, J. (2016). Starting small in assessment change: short in-class written responses. Assessment \& Evaluation in Higher Education, 41, 1114-1127. https://doi.org/10.1080/02602938.2015.1068272

El-Maaddawy, T., \& Deneen, C.C. (2017). Outcomes-based assessment and learning: Trialling change in a postgraduate civil engineering course. Journal of University Teaching \& Learning Practice.

Fisher, P., Zeligman, D., \& Fairweather, J. (2005). Self-assessed student learning outcomes in an engineering service course. International Journal of Engineering Education, 21, 446-456.

Gao, F., Yao, L., Yao, Y., Cui, X., \& Zhang, D. (2009). Research on the teaching method for computer network course. IFCSTA 2009 Proceedings - 2009 International Forum on Computer Science-Technology and Applications (2, 414-416). https://doi.org/10.1109/IFCSTA.2009.223

Gao, Y., Peng, J., Yin, Y., Hei, X., \& Wang, X. (2018). Improving a Software/Hardware Integrated Computer Networking Laboratory Course. 2018 IEEE International Conference on Teaching, Assessment, and Learning for Engineering (TALE) (1189-1192). https://doi.org/10.1109/TALE.2018.8615416

Gurgel, P.H., Barbosa, E.F., \& Branco, K.R. (2013). Teaching computer networks: A practical approach using virtualization tools. Proceedings - Frontiers in Education Conference, FIE (1021-1026). https://doi.org/10.1109/FIE.2013.6684981

Janitor, J., Jakab, F., \& Kniewald, K. (2010). Visual learning tools for teaching/learning computer networks: Cisco Networking Academy and packet tracer. 6th International Conference on Networking and Services, ICNS 2010, Includes LMPCNA 2010; INTENSIVE 2010 (351-355).

https://doi.org/10.1109/ICNS.2010.55

Jayalaxmi, G.N., Suvarna, G.K., \& Sujatha, C. (2012). Outcome based approach to teaching, learning and assessment in computer networks: An experience. AICERA 2012 - Annual International Conference on Emerging Research Areas: Innovative Practices and Future Trends (2-7). https://doi.org/10.1109/AICERA.2012.6306743

Liu, Y., Zhang, L., \& Jiao, F. (2009). Teaching computer networking experiment in the realistic network laboratory. Proceedings - 2009 International Conference on Computational Intelligence and Software Engineering, CiSE 2009 (9-12). https:/ / doi.org/10.1109/CISE.2009.5363867

Mitchell, G.G., \& Delaney, J.D. (2004). An assessment strategy to determine learning outcomes in a software engineering problem-based learning course. International Journal of Engineering Education, 20 , 494-502.

Sarkar, N.I. (2006). Using Practical Laboratory Exercises. IEEE Transactions on Education, 49, $285-291$. https://doi.org/10.1109/TE.2006.873967

Software Radio Systems Limited (2021). srsR AN Your own mobile network. Available at: https://www.srslte.com/

Vijayalakshmi, M., Desai, P., \& Raikar, M.M. (2017). Packet tracer simulation tool as pedagogy to enhance learning of computer network concepts. Proceedings - 2016 IEEE 4th International Conference on MOOCs, Innovation and Technology in Education, MITE 2016 (71-76). https://doi.org/10.1109/MITE.2016.024

Vivar, M.A., \& Magna, A.R. (2008). Design, implementation and use of a remote network lab as an aid to support teaching computer network. 3rd International Conference on Digital Information Management, ICDIM 2008 (905-909). https:// doi.org/10.1109/ICDIM.2008.4746847 
Yu, L., \& Zhang, W. (2010). Failure case study: An instructive method for teaching computer network engineering. ICCSE 2010 - 5th International Conference on Computer Science and Education, Final Program and Book of Abstracts (296-299). https://doi.org/10.1109/ICCSE.2010.5593630

Zhamanov, A., \& Sakhiyeva, Z. (2015). Implementing flipped classroom and gamification teaching methods into computer networks subject, by using cisco networking academy. 2015 Twelve International Conference on Electronics Computer and Computation (ICECCO) (1-4). https://doi.org/10.1109/ICECCO.2015.7416890

Zhang, X., \& Xiong, Z. (2013). Research on teaching methods of operating system in local university. Lecture Notes in Electrical Engineering (LNEE) (163, 1763-1768). https://doi.org/10.1007/978-1-4614-3872-4_225

Published by OmniaScience (www.omniascience.com)

Journal of Technology and Science Education, 2021 (www.jotse.org)

\section{(c) $(1) \Theta$}

Article's contents are provided on an Attribution-Non Commercial 4.0 Creative commons International License.

Readers are allowed to copy, distribute and communicate article's contents, provided the author's and JOTSE

journal's names are included. It must not be used for commercial purposes. To see the complete licence contents, please visit https://creativecommons.org/licenses/by-nc/4.0/. 